\title{
Treatment of patients with refractory metastatic cancer according to molecular profiling on tumor tissue in the clinical routine: an interim-analysis of the ONCO-T-PROFILE project
}

\author{
Andreas Seeber ${ }^{1,2}$, Guenther Gastl ${ }^{1}$, Christian Ensinger ${ }^{3}$, Gilbert Spizzo ${ }^{4}$, Wolfgang \\ Willenbacher ${ }^{1}$, Florian Kocher ${ }^{1}$, Christoph Leitner ${ }^{1}$, Ella Willenbacher ${ }^{1}$, Arno \\ Amann ${ }^{1}$, Normann Steiner ${ }^{1}$, Wolfgang Eisterer ${ }^{1}$, Andreas Voss ${ }^{5}$, Kenneth Russell ${ }^{5}$ \\ and Heinz Zwierzina ${ }^{1}$ \\ ${ }^{1}$ Department of Haematoloy and Oncology, Innsbruck Medical University, Austria \\ ${ }^{2}$ Laboratory for Experimental Oncogenomics, Tyrolean Cancer Research Institute, Austria \\ 3 Department of Pathology, Innsbruck Medical University, Austria \\ ${ }^{4}$ Haematooncological Day Hospital, Hospital of Merano, Italy \\ ${ }^{5}$ Caris Life Sciences, Basel, Switzerland \\ Correspondence to: Heinz Zwierzina, email: heinz.zwierzina@i-med.ac.at \\ Keywords: molecular profiling, cancer, personalized medicine, caris life sciences, next generation sequencing \\ Received: May 20, $2016 \quad$ Accepted: October 25, $2016 \quad$ Published: October 28, 2016
}

\section{ABSTRACT}

Introduction: Patients with refractory metastatic cancer have been shown to benefit from molecular profiling of tumor tissue. The ONCO-T-PROFILE project was launched in March 2014 at the Innsbruck Medical University. Within 2 years our project aims to recruit 110 patients with stage IV cancer refractory to standard therapy. Our data presented here are based on an interim-analysis.

Methods: Tumor tissue specimens were submitted for molecular profiling to the certified laboratory (Caris Life Science, USA). Druggable tumor targets were selected based on biomarker status to agents with potential clinical benefit. Clinical benefit was defined as a PFS ratio (=PFS upon treatment according to the molecular profile/ PFS upon the last prior therapy) $\geq 1.3$.

Results: As of April 2015, tumors from 50 patients have been molecularly profiled and one or more targets were detectable in 48 specimens $(98 \%)$. So far, $19(38 \%)$ patients have been treated according to their molecular tumor profile. To date, 8 (42\%) patients have reached a PFS ratio of $\geq 1.3$.

Conclusions: We could show that molecular profiling is feasible in the clinical routine. A proportion of patients might benefit from an individualized treatment approach based on molecular profiling. As a result, we will proceed to enroll patients in ONCO-T-PROFILE.

\section{INTRODUCTION}

The standard treatment for metastatic cancer patients is based on the histopathology of tumor tissues. However, in the last years a new wave of knowledge about the genomic and molecular structure of cancer cells has entered routine clinical practice. This has led to the approval of many drugs capable to target specific molecular alterations in a certain tumor, e.g., gefitinib in EGFR-mutated non-small cell lung cancer [1] or trastuzumab in HER2-amplified or overexpressed breast cancer [2]. The Cancer Genome Atlas Network revealed that specific genomic mutations and alterations are expressed not only in one certain histopathological defined tumor (i.e. BRAF in melanoma) but also in tumors deriving from other origins [3, 4]. This led to the consideration to abandon "classic" organ-specific histopathological analyses and to diagnose and treat patients according to their molecular profile [5].

In 2006, a feasibility analysis showed that by using immunohistochemistry (IHC) and oligonucleotide microarrays, a druggable target could be found in $98 \%$ 
of profiled cases. Therefore, a pilot-study was conducted to analyze the effect of targeted therapy according to the molecular profile of a metastatic tumor. In total, 86 patients with different refractory metastatic cancers showed a response rate of $27 \%$ with a $30 \%$ prolonged PFS (ePFS > 1.3) compared with the PFS after previous treatment [6]. A further study investigated the survival benefit of molecular characterization in 25 breast cancer patients with more than 3 prior treatment lines. In total, $44 \%(n=11)$ showed a PFS ratio of $>1.3$ [7].

The first randomized trial to investigate the value of treatment according to molecular profiling was the SHIVA trial. This phase II trial enrolled 195 patients with any kind of metastatic tumors refractory to standard treatments and randomly assigned to treatment according to molecular profiling or physicians' choice. Surprisingly, no advantage in terms of survival could be shown for patients treated with regimens based on molecular phenotyping [8]. The majority of treatment associations $(74 \%)$ in this study was not based on clinical data but followed hypotheses based on preclinical data.

In the last few years so-called "basket" trials were designed to target patients with a specific genomic alteration independent of the histology-based diagnosis. A phase II trial investigated the effect of vemurafenib in BRAF-mutated non-melanoma tumors. The response rate was $42 \%$ and the median PFS was calculated at 7.3 months. Interestingly, the activity was stronger in some entities, such as non-small cell lung cancer, but lower in others, such as ovarian or colorectal cancer [9]. It was shown later, that in colorectal cancer combination therapies of vemurafenib or dabrafenib with an EGFR directed monoclonal antibody [10] or with a MEK inhibitor [11] could successfully be used to treat patients with a BRAF mutation. These data show that the effect of molecularly-based treatment allocation needs further refinement. For this reason we established the "ONCOT-Profile" project. The aim of this project is to treat 110 patients with different refractory tumors according to their molecular profile analyzed by methods such as nextgeneration sequencing (NGS) or immunohistochemistry (IHC). Here, we present the data of the interim analysis.

\section{PATIENTS AND METHODS}

\section{The ONCO-T-PROFILE project}

ONCO-T-PROFILE was initiated in March 2014 at the Department of Haematology and Oncology of the Innsbruck Medical University. The aim is to treat 110 patients with advanced solid tumors with no further standard antineoplastic treatment options available, in a personalized manner. Therefore, after obtaining informed consent, a mandatory biopsy or an archieved sample from the resection of the tumor is collected and sent to a certified laboratory (Caris Life Sciences, Phoenix, AZ, USA) where multi-modal molecular profiling is performed. After approximately two weeks, a detailed case report with illustration of mutations and potential targetable structures is sent back to the investigator site in Innsbruck, Austria. The results of this molecular profiling are discussed among the treating physicians, Caris Life Sciences and an expert panel of the ONCO-T-PROFILE team. According to blood tests and performance status of the patient, a personalized therapy approach may be

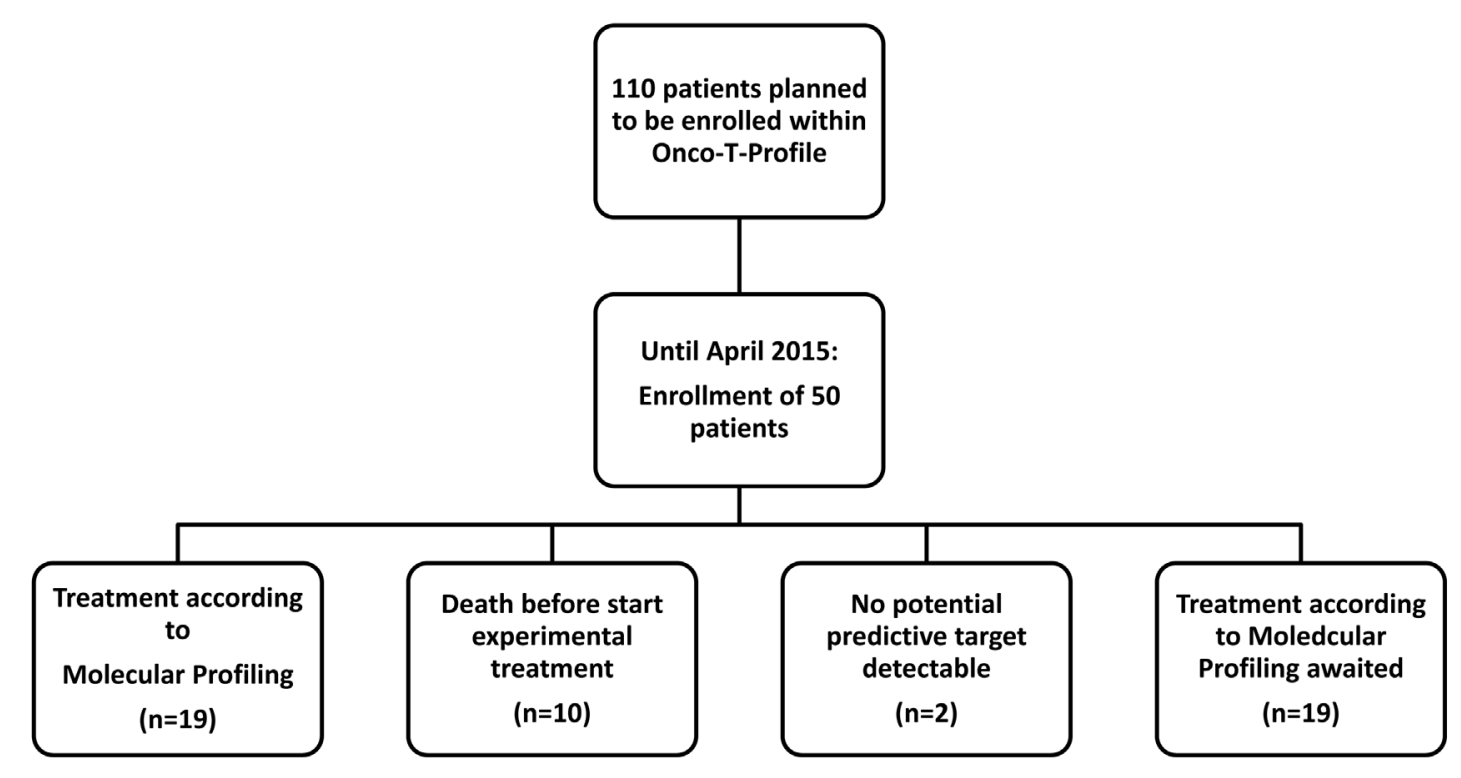

Figure 1: Consort diagram of the interim analysis of the ONCO-T-PROFILE project 
Table 1: Clinical characteristics of the first 50 patients enrolled in the ONCOT-PROFILE project.

\begin{tabular}{|l|c|c|}
\hline \multicolumn{1}{|c|}{ Baseline Characteristics } & No. of Patients & Percentage \\
\hline Total patients & 50 & 100 \\
\hline Age, years & & \\
\hline Mean & 57 & \\
\hline Range & $21-83$ & \\
\hline Sex & 32 & 64 \\
\hline Female & 18 & 36 \\
\hline Male & & \\
\hline ECOG PS & 17 & 34 \\
\hline 0 & 21 & 42 \\
\hline 1 & 12 & 24 \\
\hline 2 & & \\
\hline Tumor entity & 14 & 28 \\
\hline GI Cancer & 7 & \\
\hline CRC & 3 & \\
\hline Pancreatic & 2 & \\
\hline Gastric & 2 & \\
\hline CCC & 20 & \\
\hline Gynaecologic Cancer & 8 & \\
\hline Breast Cancer & 7 & \\
\hline Ovarian Cancer & 5 & \\
\hline Others & 3 & 10 \\
\hline Sarcoma & 3 & \\
\hline NET/NEC & 5 & \\
\hline Lung Cancer & 4 & \\
\hline NSCLC & 1 & \\
\hline SCLC & 5 & \\
\hline Other Malignancies & & \\
\hline
\end{tabular}

recommended by the treating physician. Two to three cycles or 2-3 months of therapy should be given before a restaging by imaging is performed.

The objective of this project is to compare the progression-free survival (PFS) obtained by the experimental therapy with the PFS of the last treatment on which the same patient experienced a progress. As such, each patient is her/his own historical control.

\section{Patient`s selection}

Patients older than 18 years with a histologically confirmed metastatic and recurrent solid tumor that failed standard treatment are eligible for this project. Formalinfixed paraffin-embedded (FFPE) tumor material to perform molecular profiling must be available. Patients with an Eastern Cooperative Oncology Group (ECOG) Performance status between 0 and 2 are allowed to participate. Furthermore, a life expectancy of more than 3 months, adequate liver, renal and bone marrow functions, and a written informed consent are required.

\section{Molecular profiling}

Molecular Profiling (MP) is performed on FFPE specimens using the "Caris Molecular Intelligence" (CMI) service. For that, multiple different standard platforms and methods, including next-generation sequencing (NGS), immunohistochemistry (IHC) and in-situ hybridizations (FISH/CISH), are used. The type of analyses performed and the specific biomarkers tested depended on the amount of tissue sample available.

IHC analysis was performed on formalin-fixed paraffin-embedded (FFPE) tumor samples using commercially available certified detection kits, automated staining techniques including BenchMark XT (Ventana Medical Systems, Inc., Tucson, AZ) and Autostainer Link 48 (Dako North America, Inc., Carpinteria, CA), and commercially available antibodies.

FISH and CISH was used to evaluate HER2/neu [HER2/CEP17 probe], EGFR [EGFR/CEP7 probe], and cMET [cMET/CEP7 probe] (Vysis PathVysion FISH assay, Abbott Laboratories, Abbott Park, IL). HER2/ neu and cMET status were evaluated by CISH using the 
Table 2: Clinical characteristics and administered therapies according to the molecular profiling of the first 19 patients.

\begin{tabular}{|c|c|c|c|c|c|c|c|}
\hline Patient ID & $\begin{array}{l}\text { Age } \\
\text { [years] }\end{array}$ & ECOG PS & Cancer Type & 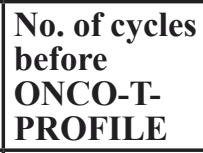 & Experimental Regimen & $\begin{array}{l}\text { PFS } v s . \\
\text { ePFS [days] }\end{array}$ & $\begin{array}{l}\text { PFS } \\
\text { Ratio > } \\
1.3\end{array}$ \\
\hline P1 & 44 & 2 & $\mathrm{CRC}$ & 8 & $\begin{array}{ll}\text { nab-Paclitaxel } \\
\text { Gemcitabine* }\end{array}$ & 86 vs. 56 & No \\
\hline $\mathrm{P} 2$ & 65 & 0 & $\mathrm{CRC}$ & 7 & Doxorubicin* & 98 vs. 62 & No \\
\hline P4 & 68 & 0 & Sarcoma, NOS & 4 & Paclitaxel + Gemcitabine & 44 vs. 237 & Yes \\
\hline P3 & 47 & 2 & $\begin{array}{l}\text { Adrenocortical } \\
\text { Carcinoma }\end{array}$ & 5 & nab-Paclitaxel* & 56 vs. 30 & No \\
\hline P15 & 37 & 1 & Breast Cancer & 8 & Exemestane + Everolimus & 184 vs. 249 & Yes \\
\hline P5 & 67 & 1 & Liposarcoma & 5 & Gemcitabine & 269 vs. 93 & No \\
\hline P18 & 64 & 0 & Breast Cancer & 5 & Carboplatin + Gemcitabine & 220 vs. 250 & No \\
\hline P7 & 46 & 2 & $\begin{array}{l}\text { Pancreatic } \\
\text { Cancer }\end{array}$ & 2 & Regorafenib* & 36 vs. 56 & No \\
\hline P8 & 55 & 2 & SCLC & 5 & Irinotecan* & 54 vs. 68 & No \\
\hline P9 & 67 & 1 & NET & 2 & Topotecan & 89 vs. 194 & Yes \\
\hline P11 & 69 & 0 & NSCLC & 4 & Gemcitabine & 62 vs. 135 & Yes \\
\hline P6 & 83 & 0 & $\begin{array}{l}\text { Endometrial } \\
\text { Carcinoma } \\
\end{array}$ & 2 & Liposomal Doxorubicin & 243 vs. 74 & No \\
\hline $\mathrm{P} 12$ & 49 & 1 & Gastric Cancer & 3 & Epirubicin + Docetaxel & 204 vs. 100 & No \\
\hline $\mathrm{P} 10$ & 51 & 0 & Breast Cancer & 2 & Exemestane + Everolimus & 17 vs. 124 & Yes \\
\hline P16 & 64 & 1 & $\begin{array}{l}\text { Endometrial } \\
\text { Carcinoma }\end{array}$ & 2 & Liposomal Doxorubicin & 71 vs. 156 & Yes \\
\hline P14 & 45 & 0 & Breast Cancer & 3 & Exemestane + Everolimus & 242 vs. 325 & Yes \\
\hline P17 & 25 & 0 & $\begin{array}{l}\text { Ovarian } \\
\text { Cancer }\end{array}$ & 1 & Everolimus & 83 vs. 156 & Yes \\
\hline P19 & 67 & 2 & $\mathrm{CCC}$ & 5 & nab-Paclitaxel* & 264 vs. 57 & No \\
\hline P13 & 57 & 1 & CRC & 3 & Regorafenib & 223 vs. 176 & No \\
\hline
\end{tabular}

Abbreviations: CRC: colorectal cancer, NOS: not otherwise specified, SCLC: small cell lung cancer, NSCLC: non-small cell lung cancer, NET: neuroendocrine tumor, CCC: cholangiocellular-carcinoma. ${ }^{*}$ : therapies which are not used in the respective indication

INFORM HER2 Dual ISH DNA Probe Cocktail, and the Chromosome 7 DIG Probe (Ventana Medical Systems, Inc., Tucson, AZ). The same scoring system was applied as for FISH. Either the absolute gene copy number in tumor cells or a gene: CEP17 signal ratio was used to score results in both methods.

HER2 CISH test was carried out using the INFORM DUAL HER2 ISH Assay (Ventana). Control was CEP17. Cutoff was HER2/CEP17 ratio $>=2.0$. cMET CISH was carried out using a probe specific for cMET - pericentromeric region of chromosome 7 (Ventana).
Positivity for increased gene copy number for cMET CISH has been defined as mean of $\geq 5$ copies of MET gene per cell in NSCLC, because the gene copy number threshold for other tumor types has not been determined. TOP2A CISH was carried out using a probe specific for TOP2A - pericentromeric region of chromosome 17 (Ventana). Control was CEP17. Cutoff was TOP2A/CEP17 ratio $>$ $=2.0$ or the presence of the mean of $\geq 6$ copies of the TOP2A in cancer cells. EGFR CISH was carried out using a probe specific for EGFR - pericentromeric region of chromosome 7 (Ventana). 
Direct NGS analysis was performed on genomic DNA isolated from FFPE tumor samples using the MiSeq platform (Illumina, Inc., San Diego, CA). Specific regions of 45 genes of the genome were amplified using the TruSeq Amplicon Cancer Panel (Illumina, Inc., San Diego, CA). Mutation analysis by Sanger sequencing included selected regions of BRAF, KRAS, cKIT, EGFR, and PIK3CA genes and was performed by using M13linked PCR primers designed to amplify target sequences. The depth of coverage was $>1000 \mathrm{X}$. Depth of coverage in DNA sequencing refers to the number of times a nucleotide is read/analyzed during the sequencing process. Coverage is the average number of reads representing a given nucleotide in the reconstructed sequence. 100\% of NGS samples were microdissected after pathologist identification of tumor cells.

\section{Statistics}

According to the study of Von Hoff and colleagues [6] a PFS ratio of $\geq 1.3$ is warranted to display a clinically relevant benefit of experimental therapy. Progression free survival $(=$ PFS) was defined as time of treatment start to date of tumor progression. The PFS ratio was defined as PFS under molecular guided therapy / previous PFS on which patient progressed. To allow for benchmarking our results we decided on using the same threshold value defining a positive outcome.

\section{RESULTS}

\section{Patient population}

From March 2014 until April 2015, 50 patients with refractory solid cancer were enrolled in our ONCO-TPROFILE project. As illustrated in Figure 1, so far, 19 patients were treated according to molecular profiling. Only in 2 of 50 patients (4\%) we were not able to detect any potential targetable alteration. Twenty-nine patients (58\%) are currently on standard therapy with already performed tumor profiling that will allow a potential switch to experimental treatment if their performance status and blood tests allow. Of the 50 patients enrolled within the ONCO-T-PROFILE program so far, breast cancer $(n=8,16 \%)$ was the most dominant tumor type,

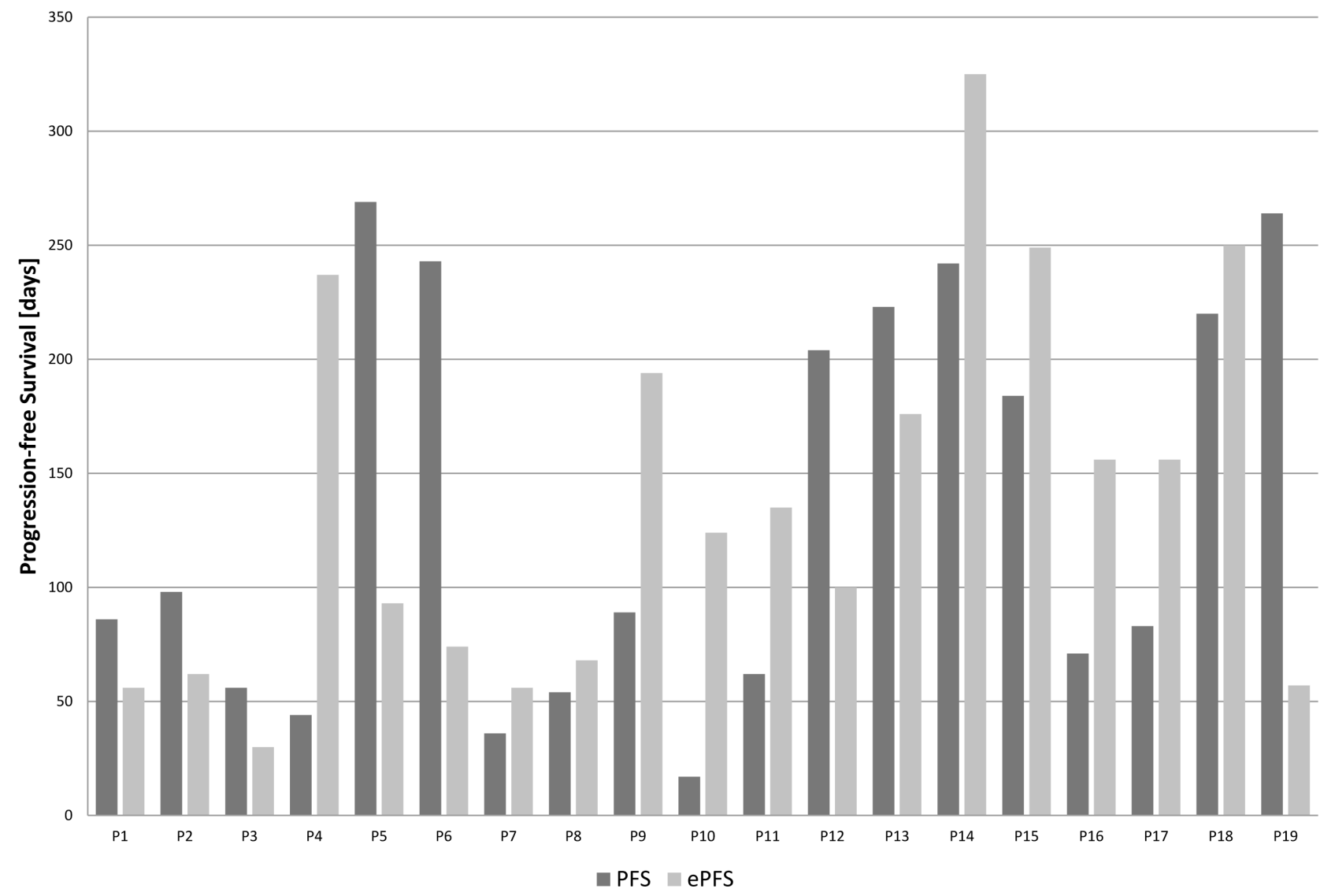

Figure 2: Progression-free survival comparison between the experimental treatment (ePFS; grey) according to molecular profiling and the prior therapy (PFS; black) of the first 19 patients treated within the ONCO-T-PROFILE project. 
followed by colorectal and ovarian cancer (both $n=7$, $14 \%)$. Five patients (10\%) suffered from lung cancer (nonsmall cell lung cancer: $n=4,8 \%$; small cell lung cancer: $n=1,2 \%)$. The other patients suffered from pancreatic cancer $(n=3 ; 6 \%)$, sarcoma $(n=3 ; 6 \%)$, neuroendocrine tumor/carcinoma $(n=3 ; 6 \%)$, gastric cancer $(n=2 ; 4 \%)$ and cholangiocellular-carcinoma $(n=2,4 \%)$. The rest of the patients had other tumors, such as adrenocortical or endometrial carcinoma (in total 10 patients, 20\%; see table 1). The mean age of treated patients was 57 years (range: $21-83$ years) and the majority was female $(n=32$, $64 \%$ ). Seventeen patients $(34 \%)$ had a good ECOG PS of 0,21 patients $(42 \%)$ had an ECOG PS of 1 and 12 patients (24\%) had an ECOG PS of 2.

\section{The effect of molecularly based treatment}

In Table 2 the clinical data of the 19 patients treated with experimental regimens according to the molecular profiling results are listed. Mean age was 76 years (range: 25-83 years) and patients had received between 1 and 8 treatment lines prior to molecular guided therapy. Eight patients had an ECOG PS of 0, 6 patients an ECOG PS of 1 , and further 5 patients an ECOG PS of 2.

In Table 2 and Figure 2 the effect of the experimental treatment according to the molecular characterization of the tumor samples is displayed. Of the first 19 patients treated within ONCO-T-PROFILE, 8 patients (42\%) had a PFS ratio $>1.3$ (range: 0,22 to 7,29 ; median: 1,26 ).

\section{Genomic alterations detected by next-generations sequencing}

In the 50 patients analyzed by next-generation sequencing (NGS) 59 mutations were detected (Figure 3). In 12 patients $(24 \%)$, no relevant pathological alteration could be found by NGS. The most common detectable gene mutation was located in TP53 $(n=14,28 \%)$ followed by BRCA 2 mutation $(n=10,20 \%)$. APC and K-RAS mutations, typically colorectal cancer-associated, were seen in 6 cases $(12 \%)$, respectively. In two patients (4\%) BRCA 1, PIK3CA and HER2 alterations, which are commonly found in breast cancer, were observed. In $4 \%$ $(n=2)$ of patients ErbB4 and FBXW7 mutations were detected. Rare mutations such as AKT1 or JAK3 were seen in one patient $(2 \%)$, respectively.

\section{DISCUSSION}

To the best of our knowledge, ONCO-T-PROFILE is the first ongoing project to investigate molecular-based targeted therapy in patients with refractory solid tumors in the clinical routine. In this interim-analysis, we describe the first 50 molecularly typed patients enrolled at the Innsbruck Medical University of whom 19 patients were treated according to their molecular profile. In 48 of 50 patients at least one potential target could be found. Up to April 2015 we have treated 19 of 50 patients using drugs which were predicted to be of potential benefit to the patient. The other 29 patients are still on standard

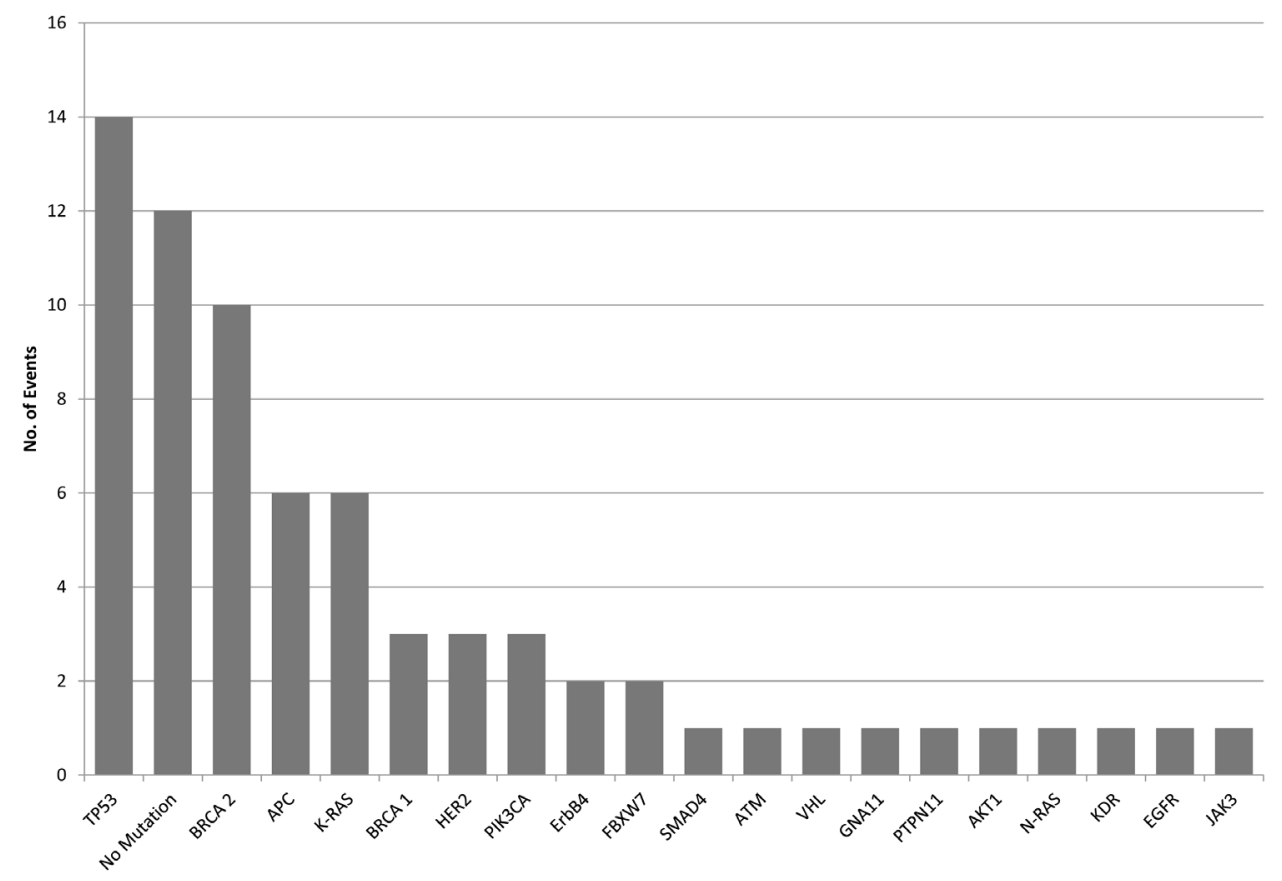

Figure 3: Mutational aberrations detected by next-generation sequencing of the 50 patients within the ONCO-TPROFILE project. 
treatment and planned to receive therapy according to molecular profiling at the time of progression, presupposed performance status will allow. This personalized approach resulted in $42 \%$ of patients achieving a $30 \%$-prolonged progression-free survival (PFS) compared to the previous PFS of the last standard therapy. In these patients, who were treated with drugs that would not otherwise have been chosen, even prolonged responses could be observed. This is in line with the results obtained by the group of Von Hoff D et al which performed a study investigating the clinical effect of personalized therapy using IHC, FISH and oligonucleotide microarray in a heavily pretreated mixed patient cohort. They found a prolonged PFS ratio in $27 \%$ of patients who received experimental treatment. Although our study used more modern and more sensitive methods (such as next-generation sequencing) compared to Von Hoff D et al, we could not find a higher rate of potentially druggable targets (96\% vs. 98\%) [6].

In recent years, personalized therapy has got into the focus of clinical research since different collaborative groups such as the Cancer Genome Atlas Network have not only found mutations but also overexpression of glycoproteins on the surface of tumor cells [12-14]. This led to the development of novel agents targeting specific structures on the tumor membrane, such as EGFR or HER2 [1;2]. In our cohort we used next-generation sequencing (NGS) to detect the most common mutated genes. The majority of patients enrolled had breast and colorectal cancer, so that TP53 mutation was the most common observed mutation followed by BRCA-2, K-RAS and APC.

In this interims analysis we wanted to analyze whether such a profiling was feasible in the clinical routine or not. We could demonstrate that molecular profiling in patients with an ECOG PS 0-2 is practicable and might result in a substantial clinical benefit. Ten patients $(20 \%)$ were already lost prior to experimental treatment due to worsening condition. Notably, 7 of those 10 patients were in an ECOG PS 2 and other 3 patients in an ECOG PS 1. The results so far are not matured enough, but we hypothesize that mainly patients in a good performance status are able to gain a benefit of such molecularly based treatments.

The impact of molecular profiling in metastatic tumor patients remains controversial. The only randomized phase II trial evaluating treatment according to molecular profiling compared to physicians ' choice failed to show prolonged survival in patients treated according to molecular phenotyping [8]. However, we and others provide first data reflecting the potential benefit of a personalized approach in selected patients $[6 ; 7]$. These controversial data might be explained due to a different approach to molecular profiling. In the SHIVA trial analysis was mainly performed by nextgeneration sequencing, gene copy number alterations and immunohistochemistry was used for hormone receptor status and to confirm any deletions or amplifications. In the certified laboratory of Caris Life Sciences, where the molecular profiling of our and other studies [6;7] was performed, In the molecular profiling used for ONCO-TPROFILE a wider panel of potential predictive markers was used. In addition, the predictive treatment associations in CMI are based on published clinical literature and are regularly updated to provide the most recent information. The treatment algorithms used in the SHIVA study were fixed and the majority was based on preclinical data or mechanistic associations. As such, we and others had more proteins available to target with specific treatment. Moreover, the end point of the SHIVA study was progression-free survival (PFS) and not the ratio between experimental PFS and the PFS on the last therapy line, as we used here. These data are missing from SHIVA but will be presented soon [15].

In conclusion, this interim-analysis of the ONCOT-PROFILE project shows the feasibility of molecular profiling in patients with advanced solid tumors refractory to standard treatments in the daily routine. A subset of patients whom underwent experimental therapy showed a prolonged PFS compared to the PFS of their previous treatment line, confirming a potential benefit of personalized targeted therapy. A complete analysis of the 110 patients within the ONCO-T-PROFILE is expected in 2017. Furthermore, due to the feasibility of molecular profiling we will broaden our spectrum of eligible malignancies comprising haematologic diseases such as multiple myeloma.

\section{CONFLICTS OF INTEREST}

AS, GG, HZ served as consultant and were in the advisory board of Caris Life Sciences.

\section{REFERENCES}

1. Maemondo M, Inoue A, Kobayashi K, Sugawara S, Oizumi S, Isobe H, Gemma A, Harada M, Yoshizawa H, Kinoshita I, Fujita Y, Okinaga S, Hirano H, et al. Gefitinib or chemotherapy for non-small-cell lung cancer with mutated EGFR. N Engl J Med. 2010; 362: 2380-2388.

2. Burstein HJ, Kuter I, Campos SM, Gelman RS, Tribou L, Parker LM, Manola J, Younger J, Matulonis U, Bunnell CA, Partridge AH, Richardson PG, Clarke K, et al. Clinical activity of trastuzumab and vinorelbine in women with HER2-overexpressing metastatic breast cancer. J Clin Oncol. 2001; 19: 2722-2730.

3. Kandoth C, Schultz N, Cherniack AD, Akbani R, Liu Y, Shen H, Robertson AG, Pashtan I, Shen R, Benz CC, Yau C, Laird PW, Ding L, et al. Integrated genomic characterization of endometrial carcinoma. Nature. 2013; 497: 67-73. 
4. Comprehensive molecular portraits of human breast tumours. Nature. 2012; 490: 61-70.

5. Majewski IJ, Bernards R: Taming the dragon. Genomic biomarkers to individualize the treatment of cancer. Nat Med. 2011; 17: 304-312.

6. Von Hoff DD, Stephenson JJ, Jr., Rosen P, Loesch DM, Borad MJ, Anthony S, Jameson G, Brown S, Cantafio N, Richards DA, Fitch TR, Wasserman E, Fernandez C, et al. Pilot study using molecular profiling of patients' tumors to find potential targets and select treatments for their refractory cancers. J Clin Oncol. 2010; 28: 4877-4883.

7. Jameson GS, Petricoin EF, Sachdev J, Liotta LA, Loesch DM, Anthony SP, Chadha MK, Wulfkuhle JD, Gallagher RI, Reeder KA, Pierobon M, Fulk MR, Cantafio NA, et al. A pilot study utilizing multi-omic molecular profiling to find potential targets and select individualized treatments for patients with previously treated metastatic breast cancer. Breast Cancer Res Treat. 2014; 147: 579-588.

8. Le Tourneau C, Delord JP, Goncalves A, Gavoille C, Dubot C, Isambert N, Campone M, Tredan O, Massiani MA, Mauborgne C, Armanet S, Servant N, Bieche I, et al. Molecularly targeted therapy based on tumour molecular profiling versus conventional therapy for advanced cancer (SHIVA): a multicentre, open-label, proof-of-concept, randomised, controlled phase 2 trial. Lancet Oncol. 2015; 16: $1324-1334$.

9. Hyman DM, Puzanov I, Subbiah V, Faris JE, Chau I, Blay JY, Wolf J, Raje NS, Diamond EL, Hollebecque A, Gervais
R, Elez-Fernandez ME, Italiano A, et al. Vemurafenib in Multiple Nonmelanoma Cancers with BRAF V600 Mutations. N Engl J Med. 2015; 373: 726-736.

10. Connolly K, Brungs D, Szeto E, Epstein RJ. Anticancer activity of combination targeted therapy using cetuximab plus vemurafenib for refractory BRAF (V600E)-mutant metastatic colorectal carcinoma. Curr Oncol. 2014; 21: e151-e154.

11. Corcoran RB, Atreya CE, Falchook GS, Kwak EL, Ryan DP, Bendell JC, Hamid O, Messersmith WA, Daud A, Kurzrock R, Pierobon M, Sun P, Cunningham E, et al. Combined BRAF and MEK Inhibition With Dabrafenib and Trametinib in BRAF V600-Mutant Colorectal Cancer. J Clin Oncol. 2015; 33: 4023-4031.

12. Comprehensive molecular characterization of human colon and rectal cancer. Nature. 2012; 487: 330-337.

13. El-Deiry WS, Vijayvergia N, Xiu J, Scicchitano A, Lim B, Yee NS, Harvey HA, Gatalica Z, Reddy S. Molecular profiling of 6,892 colorectal cancer samples suggests different possible treatment options specific to metastatic sites. Cancer Biol Ther. 2015; 16: 1726-1737.

14. Millis SZ, Bryant D, Basu G, Bender R, Vranic S, Gatalica Z, Vogelzang NJ. Molecular profiling of infiltrating urothelial carcinoma of bladder and nonbladder origin. Clin Genitourin Cancer. 2015; 13: e37-e49.

15. Le Tourneau C, Belin L, Paoletti X, Bieche I, Kamal M. Precision medicine: lessons learned from the SHIVA trial Authors' reply. Lancet Oncol. 2015; 16: e581-e582. 